\title{
Combining bone resorption markers and heel quantitative ultrasound to discriminate between fracture cases and controls
}

\author{
D. Nanchen • J. Cornuz $\cdot$ C. Ruffieux $\cdot$ W. Riesen • \\ P. Burckhardt $\cdot$ M. A. Krieg • \\ for the SEMOF study group
}

Received: 16 March 2008 / Accepted: 8 January 2009 / Published online: 31 January 2009

(C) International Osteoporosis Foundation and National Osteoporosis Foundation 2009

\begin{abstract}
Summary This nested case-control analysis of a Swiss ambulatory cohort of elderly women assessed the discriminatory power of urinary markers of bone resorption and heel quantitative ultrasound for non-vertebral fractures. The tests all discriminated between cases and controls, but combining the two strategies yielded no additional relevant information.

Introduction Data are limited regarding the combination of bone resorption markers and heel quantitative bone ultrasound (QUS) in the detection of women at risk for fracture. Methods In a nested case-control analysis, we studied 368 women (mean age $76.2 \pm 3.2$ years), 195 with low-trauma non-vertebral fractures and 173 without, matched for age,
\end{abstract}

D. Nanchen $(\bowtie) \cdot J$. Cornuz $\cdot$ M. A. Krieg

Department of Ambulatory Care and Community Medicine,

University of Lausanne,

44 Rue du Bugnon,

1011 Lausanne, Switzerland

e-mail: david.nanchen@chuv.ch

D. Nanchen $\cdot$ C. Ruffieux

Institute of Social and Preventive Medicine,

University of Lausanne,

17 Rue du Bugnon,

1005 Lausanne, Switzerland

W. Riesen

Institute of Clinical Chemistry and Hematology,

State Hospital of St.Gall,

Haus 20,

9007 St. Gall, Switzerland

P. Burckhardt

Bois-Cerf Clinic,

Avenue d'Ouchy 31,

1006 Lausanne, Switzerland
BMI, medical center, and follow-up duration, from a prospective study designed to predict fractures. Urinary total pyridinolines (PYD) and deoxypyridinolines (DPD) were measured by high performance liquid chromatography. All women underwent bone evaluations using Achilles+ and Sahara heel QUS.

Results Areas under the receiver operating-characteristic curve (AUC) for discriminative models of the fracture group, with $95 \%$ confidence intervals, were $0.62(0.56-$ $0.68)$ and $0.59(0.53-0.65)$ for PYD and DPD, and 0.64 (0.58-0.69) and $0.65(0.59-0.71)$ for Achilles+ and Sahara QUS, respectively. The combination of resorption markers and QUS added no significant discriminatory information to either measurement alone with an AUC of $0.66(0.60$ $0.71)$ for Achilles + with PYD and $0.68(0.62-0.73)$ for Sahara with PYD.

Conclusions Urinary bone resorption markers and QUS are equally discriminatory between non-vertebral fracture patients and controls. However, the combination of bone resorption markers and QUS is not better than either test used alone.

Keywords Bone markers - Bone ultrasound - Fracture risk · Nested case-control study $\cdot$ Osteoporosis

\section{Introduction}

Osteoporosis has been recognized as a major public health problem. In Western countries, hip fracture incidence rates among white women are the highest worldwide [1]. Since effective treatments exist to minimize fracture risk, it is essential that new screening strategies are developed to detect women at risk. Specific biochemical markers of bone turnover and ultrasonic measurements of bone have been 
proposed as risk factors that independently add information in the assessment of fracture probability.

The measurement of bone resorption using urinary biochemical markers has been studied as a clinical tool with which to predict fracture risk in postmenopausal women $[2,3]$. In prospective nested case-control analyses [4-6] and cohort studies [7-10], elevated bone resorption markers have been found to be associated with an increased risk of osteoporosis-related fractures. For the identification of high-risk individuals, combination strategies, using bone markers, and bone mineral density (BMD) have been shown to increase test specificity without any loss in sensitivity [11].

Quantitative ultrasound (QUS) of the calcaneus has been shown in many prospective studies to predict vertebral and non-vertebral fracture risk independent of BMD [12-18]. Comparing different QUS in the same population, we previously demonstrated that two heel QUS devices - the Achilles+ (GE-Lunar, Madison, WI, USA) and the Sahara (Hologic, Waltham, MA, USA) - are good predictors of hip and non-vertebral fracture risk, with no detectable difference between the two devices $[19,20]$.

However, few data exist with respect to the combination of bone resorption markers and heel QUS, in terms of identifying women at risk for fractures. To investigate this issue further, we performed nested case-control analysis of a Swiss cohort of post-menopausal women, comparing the results obtained using urinary levels of two bone resorption markers and two heel QUS devices, in women who had sustained a low-trauma hip or arm fracture versus those without fractures.

\section{Methods}

\section{Subjects}

Between the autumn of 1997 and summer of 1999, 7,609 ambulatory Swiss women, with a mean age of $75.3 \pm$ 3.1 years, were enrolled voluntarily in the Swiss Evaluation of Methods of Measuring Osteoporotic Fracture Risk (SEMOF) study [19, 21]. This prospective, multicenter study compared three different QUS devices-two measuring the heel and one the phalanges - as potential predictors of non-vertebral fracture risk. The SEMOF study was financed by the Association of Swiss Health Insurance, and the protocol was accepted by the Swiss Ethics Committee of Medical Sciences. Written informed consent was obtained from all women. Participants were recruited from official state registries and asked to visit one of the Swiss osteoporosis center certified to use the QUS devices. Women able to walk and independent in their daily activities were included; those with a hip fracture history or bilateral hip replacement were excluded. At baseline, participants underwent a face-to-face interview, during which they were asked about various osteoporosis risk factors, including personal fracture history, followed by a clinical examination that included calculation of body mass index (BMI). All women also underwent heel QUS. After the baseline visit, all women were asked to send back a second fasting sample of urine for the measurement of urinary bone markers. Details of study methods and patient characteristics have been reported previously [21, 22].

Identification of fractures

According to the protocol of the SEMOF study, information on vertebral fractures was not collected. We considered major osteoporotic fractures (excluding vertebral fractures), defined as low-trauma hip, distal forearm, or proximal humerus fractures [23]. Low-trauma fractures were defined as spontaneous or as a consequence of a fall from standing height or less. A medical report from the patient's attending physician was obtained to confirm each fracture. The validity of fracture information has been demonstrated previously [24].

Nested case-control design

To assess the predictive power of markers of bone resorption, 250 women with non-vertebral fractures and 250 controls matched for age, BMI, hospital, and follow-up duration were selected randomly and included in a nested case-control study. From the 500 selected women, 406 urine samples were qualitatively and quantitatively analyzable. Thirty-eight women were excluded because they were receiving hormone replacement therapy or antiresorptive therapy, like a bisphosphonate. Ultimately, 368 women195 fracture cases and 173 controls - were included in the analysis, with a mean age of $76.2 \pm 3.2$ years and a mean follow-up of $2.3 \pm 1.2$ years. Among the 195 fracture cases, 62 were hip fractures and 133 were fractures of the distal forearm or proximal humerus.

Urine sampling

Urinary samples were collected between the autumn of 1997 and summer of 1999. Each participant in the SEMOF cohort received a standard plastic container and instructions on how to collect a urine specimen at home. They were asked, following standard protocol, to send the fasting second morning specimen of urine to the Institute for Clinical Chemistry and Hematology (State Hospital of St. Gall). To minimize preanalysis variability, samples were stored frozen at $-20^{\circ} \mathrm{C}$, light protected, and never thawed until the analyses were done in 2004 . 
Measurement of bone markers

Two crosslinked collagen components, total urinary pyridinoline (PYD) and deoxypyridinoline (DPD), were measured by high performance liquid chromatography (HPLC). All analyses were conducted using the same commercial test kit: the BIO-RAD Pyridinium-Crosslinks by HPLC, a highly specific technique designed to isolate and detect pyridinium crosslinks in urine [25]. Briefly, a $150-\mu l$ urine sample, mixed with an internal standard, was hydrolyzed with $200 \mu \mathrm{l} \mathrm{HCL} 12 \mathrm{~N}$ over $16 \mathrm{~h}$ at $100^{\circ} \mathrm{C}$. After dilution, cold samples were applied to a cellulose column for extraction of interfering substances, and pyridinium crosslinks were eluted from the column. For HPLC analysis, separation of PYD and DPD was obtained by chromatography on a reverse phase column with isocratic elution. The elements' natural fluorescence was detected by spectrofluorometry. Quantitative determination was based upon comparisons against an external standard, measured in parallel. All results were expressed as crosslinks/creatinine $(\mathrm{pmol} / \mu \mathrm{mol})$. Mean precision performance for the BIO-RAD Pyridinium-Crosslinks test given by the manufacturer reached a percentage coefficient of variation of $6.4 \%$ for PYD and $6.6 \%$ for DPD [25].

\section{QUS}

Achilles + is a heel water-bath ultrasound system. Achilles + generates a band of frequencies from 200 to $600 \mathrm{kHz}$. It measures broadband ultrasound attenuation (BUA) expressed in $\mathrm{dB} / \mathrm{MHz}$, with a reported standardized coefficient of variation (SCV) of 5.8\%, and the speed of sound (SOS) expressed in $\mathrm{m} / \mathrm{s}$, with a SCV of $4.2 \%$ [26]. A third variable, the stiffness index (SI) with a SCV of $2.6 \%$, is calculated automatically, using the equation: $\mathrm{Si}=(0.67 \times \mathrm{BUA})+(0.28 \times \mathrm{SOS})-420$, expressed as a percentage of the values obtained by the manufacturer in a young adult population [27].

The Sahara QUS is a dry system ultrasound using an oilbased coupling gel. The frequencies of the ultrasounds range from 200 to $600 \mathrm{kHz}$. The two calculated variables are the BUA with a SCV of $4.4 \%$ and the SOS with a SCV of $4.3 \%$ [26]. An additional variable, the quantitative ultrasound index (QUI) with a SCV of 3.2\%, is calculated automatically, using the equation: $\mathrm{QUI}=0.41 \times(\mathrm{BUA}+\mathrm{SOS})-571$; The Result of this equation is expressed in dimensionless units [28].

\section{Statistical analysis}

Statistical tests used to compare fracture cases and controls for each baseline characteristic were Student's $t$ tests and Pearson's chi square tests, as appropriate. To estimate the predictive power of each test alone and of tests in combination, with respect to discriminating non-vertebral fractures, areas under the receiving operating characteristic curves (AUC) were calculated with 95\% confidence intervals $(95 \% \mathrm{CI})$. Differences in AUC of the predictive scores derived from the logistic regression with combinations of independent variables were tested statistically using the algorithm described in Delong et al. [29]. As no consensus exists about the threshold values for increased bone turnover, we studied two commonly used thresholds, the highest quartile against the three lowest and the premenopausal upper reference limit. The peak values for the premenopausal range were generated by the BIO-RAD laboratory kit and have been defined as per the Center for Disease Control and Prevention in Atlanta [25]. Similarly, because of the lack of established risk thresholds for QUS in clinical practice, we studied two different threshold values for each QUS device, the lowest quartile against the three highest, and a cutoff value that was able to detect, with a specificity of $80 \%$, women at high-risk for fracture or with known osteoporosis of the hip in the SEMOF cohort [20]. According to these thresholds, we estimated odds ratios (OR) with 95\% CI for those with high versus low bone marker levels and for those with low versus high QUS measurements. We estimated OR for serial (i.e., consecutive) combinations of the two diagnostic tests, using the 'and' logical operator. All analyses were performed using Stata 9.2 statistical software (Stata Corporation, College Station, TX, USA).

\section{Results}

Baseline characteristics of the 195 non-vertebral fracture patients and 173 controls are presented in Table 1. Mean age was $76.2 \pm 3.2$ years. Women who had sustained a nonvertebral fracture had statistically higher levels of bone resorption markers and lower QUS measurements than controls. As the prevalence of past fracture was not statistically different between fracture patients and controls, and adjusted OR for this factor were comparable to nonadjusted OR (data not shown), we did not enter this variable into the logistic model. In sub-analyses, considering women with arm fractures only, there were no statistical differences in DPD levels compared to controls (data not shown).

For each individual test-PYD, DPD, SOS, BUA, SI, and QUI - the AUC revealed an overall discriminatory capacity for the hip or arm fracture group (Table 2). Sahara QUI had the higher discriminatory power, with an AUC $(95 \% \mathrm{CI})$ of 0.65 (0.59-0.71); however, no test was statistically better than the other. As the discriminatory capacity of the different QUS parameters was not statisti- 
Table 1 Baseline characteristics of low-trauma fracture patients and controls

\begin{tabular}{|c|c|c|c|c|c|c|}
\hline & & Control group & $\begin{array}{l}\text { Hip or arm fracture } \\
\text { group }\end{array}$ & $\begin{array}{l}P \\
\text { value }^{\mathrm{a}}\end{array}$ & $\begin{array}{l}\text { Hip fracture } \\
\text { group }\end{array}$ & $\begin{array}{l}P \\
\text { value }^{\mathrm{a}}\end{array}$ \\
\hline Number & & 173 & 195 & & 62 & \\
\hline Age, years & & $76.3 \pm 3.2$ & $76.2 \pm 3.1$ & 0.78 & $76.9 \pm 3.0$ & 0.18 \\
\hline BMI, $\mathrm{kg} / \mathrm{m}^{2}$ & & $25.3 \pm 4.3$ & $25.5 \pm 4.1$ & 0.63 & $24.3 \pm 4.2$ & 0.10 \\
\hline History of past fracture & & $46.2(80)$ & $54.4(106)$ & 0.14 & $53.2(33)$ & 0.34 \\
\hline \multirow{2}{*}{$\begin{array}{l}\text { Urinary markers of bone } \\
\text { resorption }\end{array}$} & $\mathrm{PYD}, \mathrm{pmol} / \mu \mathrm{mol}$ & $73.1 \pm 23.5$ & $85.9 \pm 33.3$ & 0.00 & $99.6 \pm 36.7$ & 0.00 \\
\hline & $\mathrm{DPD}, \mathrm{pmol} / \mu \mathrm{mol}$ & $14.6 \pm 6.1$ & $16.4 \pm 7.3$ & 0.01 & $19.0 \pm 9.1$ & 0.00 \\
\hline \multirow[t]{3}{*}{ Achilles + heel ultrasound } & BUA, dB/MHz & $99.4 \pm 10.0$ & $95.1 \pm 9.4$ & 0.00 & $92.1 \pm 8.7$ & 0.00 \\
\hline & $\mathrm{SOS}, \mathrm{m} / \mathrm{s}$ & $1509.3 \pm 28.6$ & $1495.6 \pm 24.2$ & 0.00 & $1490.9 \pm 26.9$ & 0.00 \\
\hline & SI, \% & $68.9 \pm 13.4$ & $62.2 \pm 12.0$ & 0.00 & $59.0 \pm 12.1$ & 0.00 \\
\hline \multirow[t]{3}{*}{ Sahara heel ultrasound } & $\mathrm{BUA}, \mathrm{dB} / \mathrm{MHz}$ & $57.2 \pm 16.8$ & $49.1 \pm 12.7$ & 0.00 & $47.0 \pm 12.6$ & 0.00 \\
\hline & $\mathrm{SOS}, \mathrm{m} / \mathrm{s}$ & $1517.2 \pm 29.4$ & $1501.9 \pm 22.1$ & 0.00 & $1499.1 \pm 25.8$ & 0.00 \\
\hline & QUI, unit & $74.5 \pm 18.4$ & $64.9 \pm 13.7$ & 0.00 & $62.9 \pm 15.1$ & 0.00 \\
\hline
\end{tabular}

Values are reported as mean \pm standard deviation or percentage (number)

$B M I$ body mass index, $B U A$ broadband ultrasound attenuation, SOS speed of sound, SI stiffness index, $Q U I$ quantitative ultrasound index, $D P D$ deoxypyridinoline, $P Y D$ pyridinoline

${ }^{\mathrm{a}}$ Comparison between fracture patients and controls

cally different, we showed only the Achilles + SI and the Sahara QUI for combined strategies. The combinations of bone markers and QUS (Achilles+ SI or Sahara QUI) increased the AUC slightly but without statistically significant changes compared to either test alone. In subanalyses, the discriminatory power was higher for all tests when only the hip fracture subgroup was considered, with PYD exhibiting the highest AUC $(0.75 ; 0.69-0.82)$; but, again, no single test was statistically better than any other. The combination of PYD and QUS was statistically better than QUS alone to discriminate between hip fracture patients and controls; however, this combination was not better than PYD alone (Table 2 and Fig. 1). Considering the arm fracture subgroup only, PYD and DPD were not discriminatory (data not shown).

Table 3 shows OR with $95 \% \mathrm{CI}$ and the number of women exposed to fractures versus women without fractures, associated with defined cutoffs for PYD, DPD, SI, and QUI. For PYD and DPD, the peak limits were $>92.2$ and $17.8 \mathrm{pmol} / \mu \mathrm{mol}$ for the highest quartile, and $>63$ and 13.5 $\mathrm{pmol} / \mu \mathrm{mol}$ for the premenopausal range, respectively. For the Achilles+ SI and Sahara QUI, the risk thresholds were $\leq 57(\%)$ and $\leq 57.9$ (unit) according to the lowest quartile, and $\leq 59.1$ (\%) and $\leq 63$ (unit) according to Hans et al. [20], respectively. Women with levels of bone markers above the highest quartile or the upper limit of the premenopausal

Table 2 Area under the receiver operating-characteristic curve (AUC) for predictive models of law-trauma non-vertebral fractures, with $95 \%$ confidence intervals $(95 \% \mathrm{CI})$

\begin{tabular}{llrr}
\hline & & Hip or arm fracture group (95\% CI) & Hip fractures group (95\% CI) \\
\hline Urinary markers of bone resorption & PYD & $0.62(0.56-0.68)$ & $0.75(0.69-0.82)$ \\
& DPD & $0.59(0.53-0.65)$ & $0.70(0.63-0.78)$ \\
Achilles+ heel ultrasound & BUA & $0.61(0.55-0.67)$ & $0.69(0.62-0.77)$ \\
& SOS & $0.64(0.58-0.69)$ & $0.68(0.60-0.76)$ \\
Sahara heel ultrasound & SI & $0.64(0.58-0.69)$ & $0.70(0.63-0.78)$ \\
& BUA & $0.64(0.58-0.69)$ & $0.68(0.60-0.75)$ \\
Combination Achilles+ and markers & SOS & $0.65(0.60-0.71)$ & $0.70(0.62-0.78)$ \\
& QUI & $0.65(0.59-0.71)$ & $0.69(0.62-0.77)$ \\
Combination Sahara and markers & SI and PYD & $0.66(0.60-0.71)$ & $0.76^{*}(0.69-0.83)$ \\
& QUI and PYD & $0.65(0.59-0.70)$ & $0.74(0.66-0.81)$ \\
& QUI and DPD & $0.68(0.62-0.73)$ & $0.76^{* *}(0.69-0.83)$ \\
\hline
\end{tabular}

$B U A$ broadband ultrasound attenuation, SOS speed of sound, SI stiffness index, $Q U I$ quantitative ultrasound index, DPD deoxypyridinoline, $P Y D$ pyridinoline

${ }^{*} p<0.05$ compared to Achilles + SI alone, ${ }^{* *} p<0.05$ compared to Sahara QUI alone 

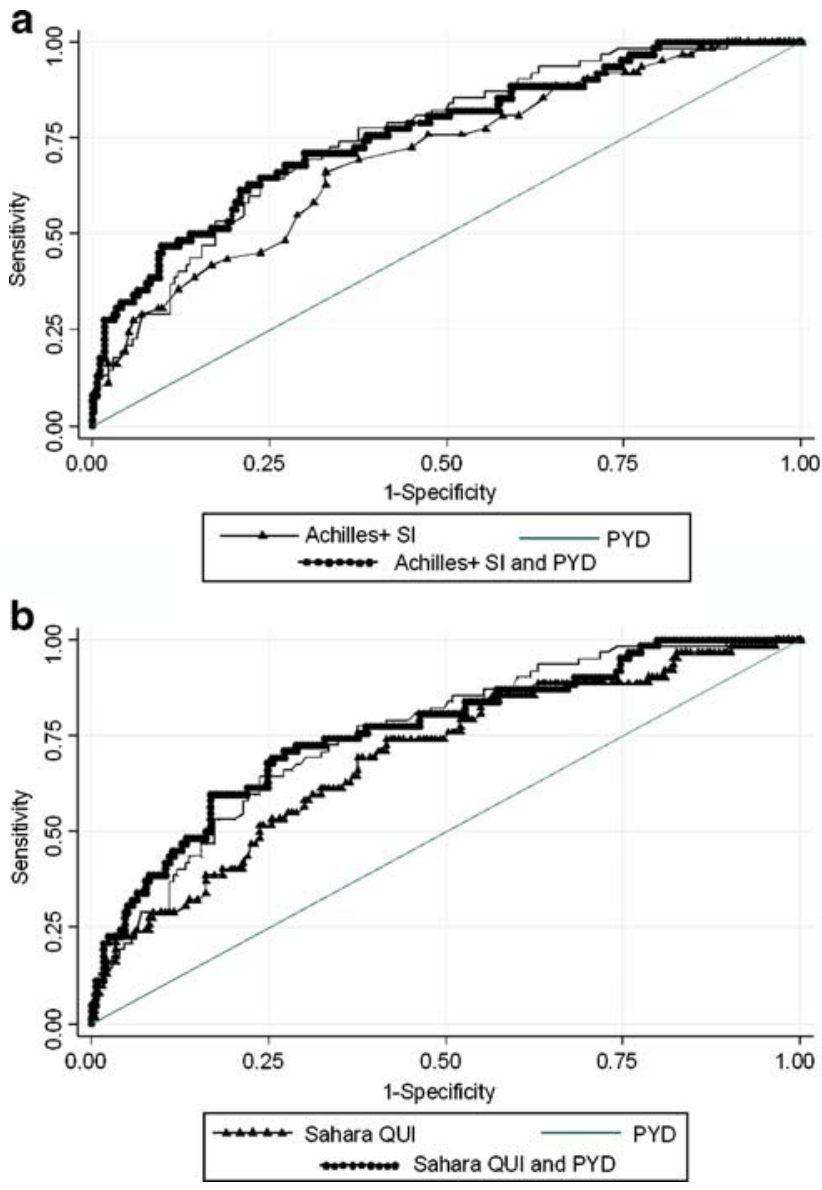

Fig. 1 Receiver operating characteristic (ROC) curves for the discrimination of hip fractures with heel quantitative ultrasounds and urinary marker of bone resorption. Achilles + SI stiffness index; PYD pyridinoline; Sahara QUI quantitative ultrasound index. a Combination of Achilles+ SI and PYD; b Combination of Sahara QUI and PYD

range were at increased risk of sustaining a non-vertebral fracture, with OR of about 2. Achilles + SI was significantly predictive only when the highest quartile was considered and was slightly less predictive than the Sahara device. Because DPD was less predictive than PYD, data on the combination of QUS and bone markers are given only for PYD. The combinations of bone markers and QUS increased slightly the prediction of fracture with both risk threshold strategies, but with larger confidence intervals. Considering the hip fracture subgroup and the quartiles as high-risk thresholds, the combination of PYD and QUS (either Achilles+ SI or Sahara QUI) had a higher predictive capacity than QUS alone; but it was not better than PYD alone (Table 3).

\section{Discussion}

In this nested case-control study including elderly women, we found that two urinary markers of bone resorption, total
PYD and total DPD, and two heel QUS, Achilles+ and Sahara, discriminate between low-trauma non-vertebral fracture patients and controls with about the same capacity. Combining the two QUS with bone resorption markers did not discriminate better than a single test between those at risk and those not at risk. Considering the hip fracture subgroup only, adding the measurement of urinary PYD to a heel QUS determination improved discriminatory performance of QUS alone, but not of PYD alone.

\section{Markers of bone resorption}

Bone resorption markers evaluate osteoclastic activity, which is the rate of bone destruction [3]. PYD and DPD are bone matrix components which stabilize the collagen molecule. They are released in the bloodstream and excreted in the urine during bone resorption. Of the different markers and measurement methods available, we used in this analysis total urinary PYD and DPD, both assessed by means of HPLC. Nowadays, direct immunoassays for PYD and DPD are widely used. The discriminatory power of PYD and DPD assessed by HPLC can be extrapolated to immunoassays currently in use, as results of immunoassays reported for total PYD and DPD are highly correlated with those measured by HPLC $(r=0.97$ for PYD ; $r=0.95$ for DPD) $[30,31]$. However, in clinical practice, the results of our study should not be extrapolated to guide the use of new serum resorption markers, like the Elecsys ${ }^{\circledR} \beta$-CrossLaps for example, as unpublished data from Roche Diagnostics have revealed only moderate correlation between urinary PYD or DPD ( $r$ about 0.7$)$. In the SEMOF cohort, serum bone markers were not measured mainly for two reasons. First, urinary sampling is a safe, non-invasive and easily performed technique. When evaluating a screening test, simplicity and acceptability for patients are important criteria, and obtaining blood samples in the SEMOF cohort was difficult as most of the participants refused blood collection. Second, because the PYD and DPD found in urine largely are derived from skeletal tissues, independent of alimentation [32], at the time the protocol for the current study was drafted in 1997, these tests were considered the most sensitive markers of bone resorption [33].

We found that elderly women with high levels of bone resorption markers were predisposed to osteoporotic fractures with an OR of about 2 . These results were consistent with previous studies, where high levels of bone markers (above the median, higher quartile, or levels above the premenopausal range) have been shown to contribute to fracture probability in elderly women [4-10] However, some recent reports have shown controversial results. In a prospective cohort study, Gerdhem et al. failed to identify any predictive capacity of urinary free DPD, measured by 
Table 3 Odds Ratios (OR) with 95\% confidence intervals (95\% CI) for non-vertebral fracture prediction and number $(\mathrm{N})$ of women in each model for a urinary marker of bone resorption [pyridinoline (PYD)] and two heel quantitative ultrasounds [Achilles+ stiffness index (SI) and Sahara quantitative ultrasound index (QUI)], associated with various risk thresholds

\begin{tabular}{|c|c|c|c|}
\hline $\begin{array}{l}\text { Quartile } \\
\text { High PYD }>92.2 \mathrm{pmol} / \mu \mathrm{mol} \text {; high } \mathrm{DPD}>17.8 \mathrm{pmol} / \mu \mathrm{mol} \\
\text { Low } \mathrm{SI} \leq 57 \% \text {; low } \mathrm{QUI} \leq 57.9 \text { unit }\end{array}$ & OR $(95 \% \mathrm{CI})$ & $\begin{array}{l}\text { Positive test } \\
\text { Fracture patients }(N) / \text { controls } \\
(N)\end{array}$ & $\begin{array}{l}\text { Negative test } \\
\text { Fracture patients }(N) / \text { controls } \\
(N)\end{array}$ \\
\hline
\end{tabular}

Hip or arm fracture group (195 fractures/173 controls)

High PYD

High DPD

Low Achilles + SI

Low Achilles+ SI and high PYD

Low Sahara QUI

Low Sahara QUI and high PYD

Hip fracture group (62 fractures/173 controls)

High PYD

High DPD

Low Achilles+ SI

Low Achilles+ SI and high PYD

Low Sahara QUI

Low Sahara QUI and high PYD

Defined risk threshold

High PYD $>63$ pmol $/ \mu$ mol; high DPD $>13.5 \mathrm{pmol} / \mu \mathrm{mol}$ : upper limit of premenopausal range

Low $\mathrm{SI} \leq 59.1 \%$; low QUI $\leq 63$ unit: thresholds from Hans et al. [20]

Hip or arm fracture group (195 fractures/173 controls)

High PYD

High DPD

Low Achilles+ SI

Low Achilles+ SI and high PYD

Low Sahara QUI

Low Sahara QUI and high PYD

Hip fracture group (62 fractures/173 controls)

High PYD

High DPD

Low Achilles+ SI

Low Achilles+ SI and high PYD

Low Sahara QUI

Low Sahara QUI and high PYD

$\begin{array}{lcc}2.0(1.3-3.1) & 149 / 107 & 46 / 66 \\ 1.7(1.1-2.6) & 119 / 82 & 76 / 91 \\ 1.5(1.0-2.4) & 71 / 47 & 124 / 126 \\ 1.9(1.2-3.1) & 60 / 33 & 135 / 140 \\ 2.1(1.4-3.2) & 99 / 57 & 96 / 116 \\ 2.4(1.5-3.8) & 168 / 125 & 27 / 48 \\ & & \\ 7.0(2.7-18.4) & 57 / 107 & 5 / 66 \\ 3.8(2.0-7.4) & 48 / 82 & 14 / 91 \\ 2.5(1.4-4.6) & 30 / 47 & 32 / 126 \\ 4.0(2.1-7.4) & 30 / 33 & 32 / 140 \\ 3.2(1.8-5.9) & 38 / 57 & 24 / 116 \\ 5.1(2.7-9.4) & 37 / 39 & 25 / 134\end{array}$

immunoassay, during a mean 4.6 years of follow-up for osteoporosis fractures in ambulatory elderly women [34]. Examining different bone turnover markers, Chen et al. found no difference between fracture cases and controls in older women and men living in residential care facilities [35]; and, in a convenient prospective cohort of elderly women with known osteoporosis and receiving treatment, Glüer et al. detected no difference between patients with or without incident vertebral fractures [36]. Mainly because of the large pre-analytical variability of bone markers and differences in the populations studied, comparing the results of these reports is fraught with difficulty.

No generally accepted threshold exists to define "high turnover rate," and most current guidelines do not give clear recommendations for the clinical use of bone resorption markers. To address this concern, we investigated different thresholds for bone markers. The upper limit of premenopausal range is a convenient and clinically applicable threshold. Values were provided by a laboratory kit, and they were less elevated than the median for PYD and about the same as the median for DPD. Thus, bone marker levels above the highest quartile identified patients at high risk for fracture and appeared to be more predictive than using the upper limit of premenopausal range because of the smaller number of patients detected. However, when interpreting the results in light of confidence intervals, there was no difference between threshold strategies. At the opposite end, a lower cutoff may be used when the goal is to rule out any increased risk of fracture. Our data show that the absence of any elevation in PYD above the premeno- 
pausal range in elderly women strongly decreases the probability of hip fracture, as only five women with a negative test sustained a hip fracture.

We also found that PYD was slightly more discriminative for the hip or arm fracture group than DPD. Physiologically, DPD is more specific for bones than PYD [37], so that a higher discriminatory capacity should have been expected. However, previous studies comparing the discriminatory power of PYD and DPD for postmenopausal osteoporosis have been inconsistent [38-40].

Combining markers of bone resorption and heel quantitative ultrasound

The use of heel QUS together with PYD added nothing to either test performed alone with respect to detecting women at risk for low-trauma non-vertebral fractures. However, in subgroup analysis, for the discrimination of hip fractures only, our results suggest that increased bone resorption might add complementary information to QUS measurements, identifying high-risk patients who were missed by QUS. To our knowledge, this improvement in the discriminatory capacity of the serial combination of QUS and bone resorption markers has not been evident in other studies. Garnero et al. found that the serial detection of C-telopeptide breakdown products (CTX) above the premenopausal range and low BMD is an improvement over the accuracy of a single test as a predictor of hip fracture, but they found no advantage of combining heel ultrasound and CTX [11]. However, in our study, performing PYD assessment alone would be as discriminative as the combination of the both PYD and QUS. Moreover, the apparent contrast between the AUC results, demonstrating no additive value when QUS and resorption markers are combined, and the large OR for those with both low QUS and high resorption markers, with quartile as threshold, might be explained by the consecutive selection of a small number participants at very high-risk of fracture, representing a small proportion of the study participants, as reflected by the large confidence intervals of OR (Table 3).

Considered the gold standard for osteoporosis assessment, BMD was not measured in the SEMOF cohort. Instead, we used different heel QUS as the reference tests for fracture prediction. However, heel QUS has been proven to predict osteoporotic fractures as well as BMD measurements in elderly women [27, 28, 41], and because QUS is relatively inexpensive, transportable, and ionizing radiation-free, there is a growing interest in its use in many countries. High levels of correlation $(r>0.8)$ have been reported between Achilles and Sahara parameters. The Achilles QUS is considered the "reference device" [26], because of its use in most of the validation studies. In our analyses, we found that Achilles+ SI was slightly less discriminatory than Sahara QUI. Cross-sectional studies comparing the two devices uncovered better results with Achilles + SI for hip fracture prediction [21, 42], but the results are inconsistent for vertebral fracture prediction [43].

Our study has limitations. Urine sample collection was standardized, so as to control for the timing of collection; nonetheless, because significant diurnal variations exist in the levels of biochemical bone markers, within-subject variability could not be avoided completely. The day-to-day variation in the urinary excretion of PYD and DPD also is reported to be a major source of within-subject variability [44], which also could affect the reproducibility of results. AUC and OR were higher in the hip fracture subgroup than in the hip or arm fracture group. This increased predictive ability could be related to more advanced osteoporosis in women who subsequently sustain a hip fracture. This difference in severity of disease may have improved the prediction power. Thus, the results we obtained within the hip fracture subgroup should not be extrapolated to justify similar screening in populations with less advanced disease. In addition, the number of hip fractures investigated was relatively low, so that the confidence intervals were broad. This reflects the need for cautious interpretation of our results when considering any individual patient. Finally, cases and controls were matched on BMI, and its effect on QUS measurements or bone turnover assessments have not been investigated. Possible overmatching could have reduced the differences observed between cases and controls.

Strategies to improve the identification of women at risk for fracture are being developed, using multiple risk factors, including clinical risk factors, QUS, and BMD measurements [45]. Our study increases the quantity of evidence supporting the relatively equal capacity of urinary bone resorption markers and heel QUS to detect elderly ambulant women at risk for low-trauma non-vertebral fractures relative to matched controls. However, the serial combination of the both tests added no complementary discriminatory information compared to either test used alone. These data, in turn, may help in the design of efficient strategies to evaluate fracture risk in elderly women.

Acknowledgements The authors are very grateful to the SEMOF group which authorized us to use their database.

Conflicts of interest None

\section{References}

1. Cummings SR, Melton LJ (2002) Epidemiology and outcomes of osteoporotic fractures. Lancet 359:1761-1767

2. Szulc P, Delmas PD (2008) Biochemical markers of bone turnover: potential use in the investigation and management of postmenopausal osteoporosis. Osteoporos Int 19:1683-1704 
3. Garnero P, Delmas PD (2004) Contribution of bone mineral density and bone turnover markers to the estimation of risk of osteoporotic fracture in postmenopausal women. J Musculoskelet \& Neuronal Interact 4:50-63

4. Garnero P, Hausherr E, Chapuy MC et al (1996) Markers of bone resorption predict hip fracture in elderly women: the EPIDOS Prospective Study. J Bone Miner Res 11:1531-1538

5. Weel AEAM, Seibel MJ, Hofman A et al (1999) Which fractures are associated with high bone resorption in elderly women: the Rotterdam study. J Bone Miner Res 14([Suppl 1]):S160 Abstract 1110

6. Chapurlat RD, Garnero P, Breart G, Meunier PJ, Delmas PD (2000) Serum type I collagen breakdown product (serum CTX) predicts hip fracture risk in elderly women: the EPIDOS study. Bone 27:283-286

7. Akesson K, Ljunghall S, Jonsson B et al (1995) Assessment of biochemical markers of bone metabolism in relation to the occurrence of fracture: a retrospective and prospective population-based study of women. J Bone Miner Res 10:1823-1829

8. Garnero P, Sornay-Rendu E, Claustrat B, Delmas PD (2000) Biochemical markers of bone turnover, endogenous hormones and the risk of fractures in postmenopausal women: the OFELY study. J Bone Miner Res 15:1526-1536

9. Ivaska KK, Gerdhem P, Akesson K et al (2007) Bone turnover markers and prediction of fracture: nine-year follow-up study of 1040 elderly women. J Bone Miner Res 22([Suppl 1]):S21 Abstract 1073

10. Melton LJ 3rd, Crowson CS, O'Fallon WM, Wahner HW, Riggs BL (2003) Relative contributions of bone density, bone turnover, and clinical risk factors to long-term fracture prediction. $\mathrm{J}$ Bone Miner Res 18:312-318

11. Garnero P, Dargent-Molina P, Hans D et al (1998) Do markers of bone resorption add to bone mineral density and ultrasonographic heel measurement for the prediction of hip fracture in elderly women? The EPIDOS prospective study. Osteoporos Int 8:563569

12. Hans D, Dargent-Molina P, Schott AM et al (1996) Ultrasonographic heel measurements to predict hip fracture in elderly women: the EPIDOS prospective study. Lancet 348:511-514

13. Bauer DC, Gluer CC, Cauley JA et al (1997) Broadband ultrasound attenuation predicts fractures strongly and independently of densitometry in older women. A prospective study. Study of osteoporotic fractures research group. Arch intern med 157:629-634

14. Stewart A, Kumar V, Reid DM (2006) Long-term fracture prediction by DXA and QUS: a 10-year prospective study. J Bone Miner Res 21:413-418

15. Pluijm SM, Graafmans WC, Bouter LM, Lips P (1999) Ultrasound measurements for the prediction of osteoporotic fractures in elderly people. Osteoporos Int 9:550-556

16. Diez-Perez A, Gonzalez-Macias J, Marin F et al (2007) Prediction of absolute risk of non-spinal fractures using clinical risk factors and heel quantitative ultrasound. Osteoporos Int 18:629-639

17. Huopio J, Kroger H, Honkanen R, Jurvelin J, Saarikoski S, Alhava E (2004) Calcaneal ultrasound predicts early postmenopausal fractures as well as axial BMD. A prospective study of 422 women. Osteoporos Int 15:190-195

18. Khaw KT, Reeve J, Luben R et al (2004) Prediction of total and hip fracture risk in men and women by quantitative ultrasound of the calcaneus: EPIC-Norfolk prospective population study. Lancet 363:197-202

19. Krieg MA, Cornuz J, Ruffieux C et al (2006) Prediction of hip fracture risk by quantitative ultrasound in more than 7000 Swiss women $>$ or $=70$ years of age: comparison of three technologically different bone ultrasound devices in the SEMOF study. J Bone Miner Res 21:1457-1463
20. Hans D, Hartl F, Krieg MA (2003) Device-specific weighted T-score for two quantitative ultrasounds: operational propositions for the management of osteoporosis for 65 years and older women in Switzerland. Osteoporos Int 14:251-258

21. Krieg MA, Cornuz J, Ruffieux C et al (2003) Comparison of three bone ultrasounds for the discrimination of subjects with and without osteoporotic fractures among 7562 elderly women. J Bone Miner Res 18:1261-1266

22. Cornuz J, Krieg MA, Burckhardt P (1999) Vorstellung der Studie "Schweizerische Evaluierung des Messmethoden des Osteoporotischen Frakturrisikos" (SEMOF). Schweiz Ärzteztg 80:349-352

23. Kanis JA, Johnell O, Oden A, Johansson H, McCloskey E (2008) FRAX and the assessment of fracture probability in men and women from the UK. Osteoporos Int 19:385-397

24. Cornuz J, Krieg MA, Sandini L et al (2003) Factors associated with the use of hormone replacement therapy among older women. Age Ageing 32:675-678

25. Bio-Rad (2002) Pyridinium-Crosslinks by HPLC, nouvelle calibration. Notice d'utilisation

26. Krieg MA, Barkmann R, Gonnelli S et al (2008) Quantitative ultrasound in the management of osteoporosis: the 2007 ISCD official positions. J Clin Densitom 11:163-187

27. Njeh CF, Blake GM (1999) Calcaneal quantitative ultrasound: Water-coupled. In: Njeh CF, Hans D, Fuerst T, Glueer C-C, Genant HK (eds) Quantitative ultrasound: assessment of osteoporosis and bone status. Martin Dunitz, London, pp 109-124

28. Cheng S, Hans D, Genant H (1999) Calcaneal quantitative ultrasound systems: Gel-coupled. In: Njeh CF, Hans D, Fuerst T, Glueer C-C, Genant HK (eds) Quantitative ultrasound: assessment of osteoporosis and bone status. Martin Dunitz, London, pp 125-144

29. DeLong ER, DeLong DM, Clarke-Pearson DL (1988) Comparing the areas under two or more correlated receiver operating characteristic curves: a nonparametric approach. Biometrics 44:837-845

30. Robins SP, Woitge H, Hesley R, Ju J, Seyedin S, Seibel MJ (1994) Direct, enzyme-linked immunoassay for urinary deoxypyridinoline as a specific marker for measuring bone resorption. J Bone Miner Res 9:1643-1649

31. Seyedin SM, Kung VT, Daniloff YN et al (1993) Immunoassay for urinary pyridinoline: the new marker of bone resorption. J Bone Miner Res 8:635-641

32. Colwell A, Russell RG, Eastell R (1993) Factors affecting the assay of urinary 3-hydroxy pyridinium crosslinks of collagen as markers of bone resorption. Eur J Clin Investig 23:341-349

33. Seibel MJ (2000) Molecular markers of bone turnover: biochemical, technical and analytical aspects. Osteoporos Int 11 Suppl 6:S18-S29

34. Gerdhem P, Ivaska KK, Alatalo SL et al (2004) Biochemical markers of bone metabolism and prediction of fracture in elderly women. J Bone Miner Res 19:386-393

35. Chen JS, Seibel MJ, Zochling J et al (2006) Calcaneal ultrasound but not bone turnover predicts fractures in vitamin $\mathrm{D}$ deficient frail elderly at high risk of falls. Calcif Tissue Int 79:37-42

36. Gluer MG, Minne HW, Gluer CC et al (2005) Prospective identification of postmenopausal osteoporotic women at high vertebral fracture risk by radiography, bone densitometry, quantitative ultrasound, and laboratory findings: results from the PIOS study. J Clin Densitom 8:386-395

37. Seibel MJ (2005) Biochemical markers of bone turnover: part I: biochemistry and variability. Clin Biochemist 26:97-122

38. Bettica P, Taylor AK, Talbot J, Moro L, Talamini R, Baylink DJ (1996) Clinical performances of galactosyl hydroxylysine, pyridinoline, and deoxypyridinoline in postmenopausal osteoporosis. J Clin Endocrinol Metab 81:542-546

39. Del Campo MT, Gonzalez-Casaus ML, Aguado P, Bernad M, Carrera F, Martinez ME (1999) Effects of age, menopause and osteoporosis on free, peptide-bound and total pyridinium crosslink excretion. Osteoporos Int 9:449-454 
40. Heiss C, Hoesel LM, Wehr U et al (2004) Vitamin $\mathrm{K}$ in combination with other biochemical markers to diagnose osteoporosis. Biomarkers 9:479-488

41. Marin F, Gonzalez-Macias J, Diez-Perez A, Palma S, DelgadoRodriguez M (2006) Relationship between bone quantitative ultrasound and fractures: a meta-analysis. J Bone Miner Res 21:1126-1135

42. Njeh CF, Hans D, Li J et al (2000) Comparison of six calcaneal quantitative ultrasound devices: precision and hip fracture discrimination. Osteoporos Int 11:1051-1062
43. Hartl F, Tyndall A, Kraenzlin M et al (2002) Discriminatory ability of quantitative ultrasound parameters and bone mineral density in a population-based sample of postmenopausal women with vertebral fractures: results of the Basel Osteoporosis Study. J Bone Miner Res 17:321-330

44. McLaren AM, Isdale AH, Whiting PH, Bird HA, Robins SP (1993) Physiological variations in the urinary excretion of pyridinium crosslinks of collagen. Br J Rheumatol 32:307-312

45. Kanis JA, Borgstrom F, De Laet C et al (2005) Assessment of fracture risk. Osteoporos Int 16:581-589 